\title{
XXXII.
}

\section{Ueber das Vorkommen und die Bedentung der Mastzellen im Nervensystem des Menschen.}

\author{
Von \\ Dr. Th. Rosenheim, \\ Assistenzarzt an Städtischen Allgem. Krankeuhause "Friedrichshain \\ (Hierzu Taf. XII.)
}

I.

Im Jahre 1877 gelang es Ehrlich (Archiv für mikroskop. Anatomie XIII. Band) durch Anwendung von Anilinfarbstoffen aus der grossen Gruppe der Wald ey er'schen Plasmazellen eine Zahl von Elementen mit bestimmtem Charakter auszusondern, die er "Mastzellen“ genannt hat. Er fand unter normalen und patbologischen Verhältnissen im Bindegewebe grosse, grob granulirte, unregelmässig gestaltete Zellen; sie lagen fast immer vereinzelt, meist in der Nähe von Gefässen. In verschiedenen Organen z. B. dem Darm war ihr Vorkommen ein constantes, in Bezug auf Zahl und Anordnung verhielten sie sich bei den gleichen Altersstufen ziemlich gleich. Ihre Erkenung gelang Ehrlich durch ihr charakteristisches Verhalten den basischen Anilinfarbstoffen gegenüber. Mit diesen färbt sich ihr unregelmässig körniges, häufig auseindergesprengtes Protoplasma, während der Kern als heller Fleck ungefärbt in der Mitte hervortritt. Mit einigen Farbstoffen (besonders den violetten) färben sie sich metachromatisch, wodurch sie noch deutlicher erkennbar werden. Im Gegensatze zu den Bacterien behalten sie äusserst resistent den Farbstoff bei Einwirkung von Säuren. Sie entstehen nach demselben Autor aus den freien Bindegewebskörpern und sie vermehren sich bej „local gesteigerten Ernährungsuständen, welche bald durch 
chronische Entzündung, bald durch Störungen (braune Induration), bald durch Neubildungen (Carcinom) hervorgerufen werden können". In Folge dieses Verhaltens bekommen sie den Namen „Yastzellen“.

Derselben Ansicht giebt Westphal (Inaugural-Dissertation, Berlin 1880) Ausdruck. Er nennt sie das Product „einer Metamorphose der unter besonders günstigen Ernährungsverhältnissen lebenden Bindegewebszellen“.

Eine solche Metamorphose hat Raudnitz (Archiv für mikroskop. Anatomie XXII. Band, 1683, S. 228) durch mucinöse Degeneration zu erklären gesucht. Diese Auffassung wurde jedoch von Nordmann (Inaugural-Dissertation, Göttingen 1884) vollkommen widerlegt, indem er zeigte, wie verschieden sich in demselben Organ Schleimzellen und Mastzellen gewissen Farbstoffen gegenüber verhalten. Auch andere Versuche, die Entstehung der Mastzellen auf einen bekannten physiologischen oder pathologischen Vorgang zurückzufübren, sind bisher missglückt. Was wir auf diesem Gebiete wissen, ist also recht spärlich und wohl im Wesentlichen durch meine Aufzählung erschöpft: und doch handelt es sich hier um sehr wichtige Elemente.

II.

Im menschlichen Nervensystem, dem centralen, wie dem peripherischen, hat man die Mastzellen bisher nicht gesucht oder nicht gefunden. In der Literatur wenigstens finde ich darüber nichts verzeichnet. Und doch kommen sie hier vor und sind gerade hier, wie mir scheint, von grosser Bedeutung.

Bei der mikroskopischen Untersuchung eines Falles von acuter infectiöser multipler Neuritis, dessen Veröffentlichung in der nächsten Zeit erfolgen soll, fand ich durch Behandlung von Nervenschnitten mit basischen Anilinfarbstoffen diese merkwürdigen Elemente zuerst. Auf Längs- und Querschnitten verschiedenartiger Nerven ergaben sich dann die unumstösslichen Beweise ihres anatomischen Charakters.

Für den Nachweis der Mastzellen bewährten sich weniger das Vesuvin und Fuchsin, nicht als ob die Mastzellen nicht mit diesen Farbstoffen zur Anschanung gebracht werden könnten, sondern einmal, weil ihre Erkennung bequemer gelingt mit den Substanzen, denen gegenüber die Zellen sich metachromatisch verhalten und zweitens, weil Fuchsin und Vesuvin zu den empfehlenswerthen Doppelfärbungen sich schlecht eignen. Ich bevorzugte deshalb das Anilinwassergentianaviolett (5-8 Minuten) in bekannter Zusammen- 
setzung und das Methylenblau (5-10 Minuten) in der Form, wie es Löffler zur Bacillenfärbung empfohlen hat (Mittheilungen aus dem Reichsgesundheitsamt 1884, Bd. II. S. 439), 30 Cbctm. concentrirter alkohol. Lösung von Methylenblau, 100 Cbctm. Kalilange (2: 10000).

Mit dem ersteren Farbstoff nehmen alle Bindegewebskerne eine hellviolette Färbung an, während das Protoplasma der Mastzellen einen tief roth-violetten Ton erhält. Bei Anwendung der zweiten Farbmischang werden die Bindegewebskerne blass grünlich, während das Protoplasma der Mastzellen tief blau erscheint. In beiden Fällen verhalten sich die zugehörigen runden Kerne, wie oben beschrieben, d. h. sie sind ungefärbt.

Für die Entfernung des überschüssigen Farbstoffes genügte absoluter Alkohol, Wollte ich in dem Präparat nur die Mastzellen gefärbt erhalten, so wandte ich Anilinwassergentianaviolett (5-8 $\mathrm{Mi}$ nuten) und dann die Gram'sche Entfärbungsmethode an, d. h. der Nervenschnitt kam aus dem Farbstoff in die Jodjodkalilösung von bekannter Constitution für 3 Minuten und wurde dann in absolutem Alkohol längere Zeit abgespült. Dann hatten die Nervenfasern, wie das Bindegewebe einen gleichmässig blassgelben Ton angenommen, von welchem die rothvioletten Mastzellen sich prächtig abhoben.

Für den Nachweis der Mastzellen im Nerven war es gleichgiltig, wie derselbe vorher behandelt worden war. Die Mastzellenreaction trat gleich klar und mit demselben Farbenton zu Tage, ob ich den Nerven frisch, nachdem er mit dem Gefriermikrotom geschnitten war, oder nach Behandlung mit Alkohol absolut. oder nach Härtung in Müller'scher Flüssigkeit untersuchte. Bemerken will ich noch, dass anch die von Ehrlich als absolut charakteristisch angegebene Reaction: Färbung $1 / 2$ Stunde lang in saurer Methylviolettlösung an mit Alkohol gehärteten Nerven erprobt wurde und nie im Stiche liess.

Um das Verbalten der Nervensubstanz bei der Anwesenheit von Mastzellen im Nerven zu studiren, wandte ich nun folgende Doppelfärbungen an:

1. Der gehärtete Schnitt kommt 10 Minuten lang in Pikrocarmin, wird dann in salzsaurem Alkohol (1:100) abgespült, hierauf bringt man ihn 5-8 Minuten in Anilinwassergentianaviolett, dann 3 Minuten in Gram'sche Lösung, endlich in absoluten Alkohol, so lange, bis das Präparat nur die Carminfärbung seben lässt. Zum Aufhellen nahm ich Cedernholzöl, die Finbettung geschah in Canadabalsam.

2. Noch empfehlenswerther erscheint mir eine Grundfärbung mit 
Carmin-A mmoniak (10-12 Minuten). Das weitere Verfahren bleibt das gleiche wie unter 1. An so bebandelten Präparaten färbt sich der Axencylinder roth, die Markscbeide bleibt ungefärbt, sämmtliche Kerne, auch die der Mastzellen werden tief roth, die Grundsubstanz des Bindegewebes blassrotb; das Protoplasma der Mastzellen aber zeigt eine prachtvoll violette Farbe und hebt sich klar ron dem übrigen Gewebe ab (siehe Fig. 1). Bei Anwendung von Pikrocarmin ging das Roth mehr in's Fleischfarbene, die fibrilläre Substanz zeigte einen Stich in's Gelbe.

Erst mit Hülfe dieser Doppelfärbungen gelang es mir festzustellen, wo die Mastzellen eigentlich liegen.

Auf Querschnitten bekam ich um die Nervenfasern herum häufig Halbmondfiguren von protoplasmatischer Körnelung, deren Lage ausserhalb oder innerhalb der Schwann'schen Scheide nur an feinsten Schnitten durch Anwendung von 1/12 OelimmersionsLinse, Ocular $0,1,3$ (Seibert) feststellbar war. Aber sicher wurde an einzelnen Stellen constatirt, dass die Körnelung, wenn auch seltener, um die in die Markschicht prominirenden Kerne der Schwann'schen Scheide, d. h. innerhalb derselben lag, dass also die Mastzellbildung von den Ranvier'schen Körpern ausgegangen war. An anderen Stellen war die Erkennung der Lage leicht, weil im Schnitt der zugehörige und tief roth gefärbte Kern der Henle'schen Schicht mit getroffen war.

In vielen Fälle füllten die violette Körnelung den Raum zwischen drei Nervenfasern in Form eines $\mathrm{N}$ a poleo $\mathrm{zh}$ utes aus, oder, zwischen benachbarten Fasern entstanden, die ganze Lücke ausfüllend, unregelmässige Figuren, der Convexität der Fasern entsprechend ausgebuchtet. Stets liessen sich die Mastzellen $n$ ur in den feinsten Verzweigungen der interstitiellen Substanz, niemals in den gröberen Maschen, geschweige denn im breiten Perineurium nachweisen; immer lag die Mastzelle der Schwann'schen Scheide unmittelbar an oder war doch nur durch eine ganz feine Fibrille von ihr getrennt.

Auf Längsschnitten konnte ich stets die ganze Mastzelle übersehen. Die wechselnden Figuren im Querschnitt fanden hier ihre Erklärung in der unregelmässigen Form des Protoplasmas: dasselbe umgab den Kern ganz oder fand sich nur an den entgegengesetzten Polen. Häufig war dasselbe in die Maschen des Bindegewebes hinein versprengt. Auch auf Längsschnitten liess sich die Zugehörigkeit der Mastzellen zur Schwann'schen Scheide und Henle'schen Schicht deutlich erkennen. 
III.

Während ich mit diesen Untersuchungen beschäftigt war, wurde meine Aufmerksamkeit durch meinen hochverehrten Lehrer, Herrn Geh. Med.-Rath Prof. Dr. Westphal, auf die Arbeit von Adamkiewicz (Sitzungsberichte der Wiener Akademie math. physik. Classe, Bd. 91, Abth. TI.) gelenkt, in der er über neu entdeckte Nervenkörperchen berichtete. Da mir mehrere der von Adamkiewicz hierher gesandten mikroskopischen Präparate zugänglich gemacht wurden, so war ich in die Lage gesetzt, mich selbst von der Richtigkeit der Angaben dieses Autors zu überzeugen. Wie erstaunt war ich aber, als ich in den Nervenkörperchen meine Mastzellen erkannte. Die Präparate, die die Nervenkörperchen enthalten sollten, waren übrigens vor einigen Monaten bereits der physiologischen Gesellschaft durch Herrn Dr. Benda vorgelegt worden, und wie ich nachträglich zu meiner Freude erfuhr, hatte auch dieser bereits die auffallende Aehnlichkeit mit Mastzellen betont.

Ich bebaupte nun, dass die von Adamkiewicz in den peripherischen Nerven Erwachsener beschriebenen Zellen keine Nervenkörperchen sind: es sind Mastzellen, wie ich sie oben ausführlich charakterisirt habe. Ich behaupte ferner, dass die von Adamkiewicz beschriebenen Zellen gar nichts direct mit der Nervensubstanz zu thun haben, da sie ausserhalb wie innerhalb der Schwann'schen Scheide nur Veränderungen der Bindegewebskörper darstellen. Ich behaupte endlich, dass die Schilderung, die Adamkiewicz von seinen Zellen entwirft, gar nicht dem Bilde entspricht, das wir unter dem Mikroskope von seinen Elementen erhalten, dass sie erheblich anders anssehen, als er sie beschreibt und zeichnet. Und davon habe ich mich an Adamkiewicz's eigenen Präparaten überzeugt, als ich sie mit genügend starker Vergrösserung musterte. Denn das scheint mir allerdings für die Entscheidung so schwieriger und wichtiger Fragen absolut nothwendig, dass man den Abbé'schen Beleuchtungsapparat und die Oelimmersionslinse zur Hülfe heranzieht. Adamkiewicz giebt selbst an, dass er sich der Trockensysteme Reichert Ocul. 3, No. 5 und No. 7 bedient hat: dies entspräche einer Vergrösserung bis 340 .

Ich halte eine solche bis ca. 1000 event. für nöthig und bediene mich bei diesen Untersuchungen der Oelimmersionslinse $1 / 12$ Ocular, 0,1 oder 3 Seibert.

Ebenso dringend nothwendig scheint mir za solchen Untersa- 
chungen die Herstellung feinster Schnitte, die ich mit Hühnereiweiss anf das Deckglas klebe und dann erst mit den verschiedenen nöthigen Stoffen in Berührung bringe. Diese Methode schützt das Präparat vor allen Zerrungen und Rissen, denen es bei der Uebertragung in die mannigfachen Flüssigkeiten ausgesetzt ist, und durch die ganz zarten Schnitte eben unbrauchbar werden.

Dieser eben geschilderten Hülfsmittel habe ich mich also für meine Arbeit bedient und, um jedem Einwand zuvorzukommen, wandte ich auch die Behandlungs- und Färbungsmethode an, wie sie Adamkiewicz wünscht: also Härtung in Müller'scher Lösung und Färbung mit Saffranin. (Sitzungsberichte der Wiener Akademie der Wiss. Bd. 89: Neue Rückenmarkstinctionen.) Es gelang mir auch dieselben Farbennüancen herauszubekommen, wie ich sie in seinen Präparaten gesehen hatte. Nur fand ich keine Nervenkörperchen, sondern Mastzellen, die sich in Saffranin ebenso charakteristisch, wie mit jedem anderen basischen Anilinfarbstoff färben d. h.: das Protoplasma nimmt die Färbung intensiv an (braungelb bis orangeroth), der runde Kern bleibt ungefärbt. Weder in meinen Präparaten, noch in denen ron Adamkiewicz sah ich spindelförmige Zellen; statt scharfbegrenzter Spindeln, wie er sie beschrieben und gezeichnet hat, fand ich unregelmässig gestaltete Zellen, deren Protoplasma oft versprengt war (s. o.).

Die Körnelung scheint Adamkiewicz an dem Protoplasma vollkommen entgangen zu sein: nach seiner Zeichnung muss das Protoplasma ganz homogene Beschaffenheit haben. Dass der Kern sich gar nicht, oder fast gar nicht im Gegensatze zu dem Protoplasma färbt, ist ihm nicht besonders aufgefallen. Und doch färben sich alle anderen Kerne im Nerven, sowohl die der Schwann'schen Scheide, als auch die des interstitiellen Gewebes prägnant mit Saffranin denn Saffranin ist eben ein basischer Anilinstoff und als solcher in erster Reihe Kernfärbungsmittel - und doch ist dieses merkwürdige Verhalten des Protoplasmas noch nie bei anderen Zellen als den sogenannten Mastzellen beobachtet worden.

Die grosse Differenz in der Deutung der von Adamkiewicz und mir gesehenen, zweifellos identischen Elemente ist schliesslich bedingt durch die verschiedene Lage, die wir den Zellen anweisen. Ich stimme in so weit mit Adamkiewicz überein, als ich mich davon überzeugt habe, dass sie innerhalb der Schwann'schen Scheide liegen können. Aber das ist durchaus nicht das häufigere. Dieselben Zellen sehen wir auch in der Henle'schen Schicht, was Adamkiewicz entgangen ist. 
IV.

Nach dem, was ich bisher gesagt habe, kann über den anatomischen Charakter der von mir im Nerven gefundenen Zellen kein Zweifel bestehen. Mit der Constatirung der Thatsache, dass Mastzellen im Nerven vorkommen, konnte ich aber natürlich meine Aufgabe nicht als erschöpft ansehen. Ich musste zunächst feststellen, ob diese Elemente unter allen Verhältnissen oder nur unter bestimmten Bedingungen im Nerven anzutreffen sind. Ich untersuchte also bei etwa 20 Individuen verschiedenen Alters, die an keiner Nervenkrankheit gestorben waren, die peripherischen Nerven und zwar nicht bloss die bequem liegenden Extremitätennerven: Peroneus, Cruralis, Uluaris a. s. w., sondern auch in mehreren Fällen Vagus und Sympathicus.

In den peripherischen Nerven eines 6 monatlichen Embryo waren die Mastzellen nicht nachweisbar. Bei Kindern von einigen Monaten bis za 5 Jahren fand ich sie niemals in den Nerven. Bei einem Knaben von 6 Jahren waren nur in zwei Schnitten je eine Mastzelle nachweisbar gewesen. Etwas häufiger sah ich sie bei einem 14 jährigen Mädchen. Bei einer 25jährigen Phthisica liessen sich in jedem Querschnitt etwa zwei nachweisen. In diesem Verhältniss fanden sie sich bis zum 45 Lebensjahre. Jenseits dieser Altersgrenze wurden sie sebr reichlich: bei einzelnen Individuen zwischen 60 und 80 Jahren ergaben sich die Nerven vollständig von ihnen durchsetzt. Es geht aus diesen Untersuchungen also hervor, dass unter physiologischen Verbältnissen die Mastzellen im Nerven im Laufe der Entwickelung jenseits des 5. Lebensjahres sich bilden, während der Blüthe des Lebens spärlich, aber doch leicht nachweisbar vorhanden sind, um im Alter ausserordent. lich zablreich zu werden. Im früben Kindesalter fehlen sie ganz.

Dieser Befund deckte sich genan mit dem, was Ehrlich bei Thieren nachweisen konnte, dass die Vermehrung der Mastzellen dem Alter proportional ist, so dass er sich mit gutem Rechte unterfing, auf Grund des Mastzellenbefundes das Lebensalter der Thiere zu bestimmen.

Was pathologische Verbältnisse anbetrifft, so fanden sich die Mastzellen überaus zahlreich in allen Nerven lange Zeit gelähmter Extremitäten (bei Hemiplegien). Da es sich hier aber ausschliesslich um ältere Individuen handelte, in deren Nerven die Mastzellen sich auch ohne dies überaus reichlich nachweisen liessen, so 
will ich hierauf keinen besonderen Werth legen; doch schienen sie mir in den gelähmten Nerven mit degenerativer Atrophie der Nervensubstanz immerhin zahlreicher zu sein als in den Nerven der entsprechenden gesunden Extremitäten.

Von viel grösserer Bedeutung war der Nachweis von Hastzellen in dem von mir bereits oben erwähnten Falle von multipler Neuritis.

Es handelte sich um ein Individuum von 35 Jahren. Der Process in den ergriffenen peripherischen Nerven war pathologisch-anatomisch charakterisirt durch Hy perämie, Hämorrhagie und Kernvermehrung in den Interstitien. In allen von der Erkrankung ergriffenen Nerven fanden sich die Mastzellen sozahlreich, wie ich sie sonst nur bei ganz alten kachelitischen Individuen zu sehen bekam. Bemerkenswerth war, dass in denjenigen Nerven, die von der Erkrankung noch nicht betroffen waren, die Mastzellen auffallend spärlich waren, spärlicher als ich sie sonst in diesem Alter zu finden pflegte. - Dafür, dass die Vermehrung der Mastzellen mit dem frischen entzündlichen Process parallel einhergegangen war, sprach auch noch ein anderer Befund, der mich die Entwickelung dieser Elemente aus den Bindegewebskörpern kennen lehrte. lch entdeckte nämlich bei der einfachen Färbung mit AnilinwasserGentianaviolett eine grosse Zahl von zelligen Elementen, die ich mir nicht anders, denn als Uebergangsformen auf dem Wege der Entwickelung der Bindegewebszellen zu Mastzellen zu deuten vermochte. Es zeigten sich in demselben Schnitte neben unzweifelhaften Mastzellen auch solche Elemente, bei denen das Protoplasma den rothvioletten Ton angenommen hatte, während der Kern von bald mehr, bald weniger hellvioletter Färbung war, also nicht ungefärbt blieb. Während der Kern der ausgebildeten Mastzellen stets kugelrund ist, zeigte sich der Kern dieser Elemente mehr länglich und damit den benachbarten Bindegewebskernen ähnlicher. Endlich fiel mir auf, dass die rothviolette protoplasmatische Körnelung um so spärlicher war, je besserderKern den Farbstoff aufgenommen hatte (siehe Fig. 2). Diese Bilder glaube ich nur so deuten zu können, dass sie die Mastzellen in verschiedenen Entwickelungsstadien vorstellen, und dass ich in diesem Falle ron multipler Neuritis in der Lage war, die Mastzellen gewissermasssen in statu nascendi zu überraschen.

V.

Ich bin deshalb auf Grund meiner Untersuchungen nur in der Archiv f. Psychiatrie, XVII. 3. Heft. 
Lage für die Mastzellen im Nerven bestätigen zu können, was EhrIich im Allgemeinen für sie in Anspruch genommen hat: Dass sie aus Bindegewebskörpern entstehen, und dass ihre Vermehrung von der Ueberernährung, wie sie das Bindegewebe z. B. im Entzündungsprocesse erfährt, abhängig ist.

Der peripherische Nerv ist zweifellos ein im Allgemeinen schlecht ernährtes Organ; die zuführenden Gefässe sind nicht sehr reichlich, das Capillarnetz ist wenig ausgebildet. Eine um so grössere Rolle spielen deshalb ja sicher für die Ernährung der Nervenfaser die Saftcanälchen des sie umziehenden Endoneuriums and entsprechend ist denn auch die Bedeutung der in diesem feinen interstitiellen Gewebe eingeschalteten Zellen. Jede Veränderung in dem Ernährungszustande des Nerven wird deshalb an diesen Elementen einen Ausdruck finden. Im entzündlichen Zustande erbält ein Nerv erheblich mehr Ernährungsmaterial, und als Ausdruck dieser reichlicheren Ernährung sehe ich die Vermehrung von Mastzellen au.

Dass die Mastzellen in den degenerativ-atrophischen Zuständen der Nerven so überaus zahlreich gefunden wurden, spricht nicht gegen die Annahme ihrer Entstehung, resp. ibrer Vermehrung durch Ueberernährung. Denn in diesen Zuständen wird, während die Zufubr die gleiche bleibt, wie früher, durch den Schwond von Nervensubstanz Ernährungsmaterial frei, das alsdann dem Bindegewebe der nächsten Nachbarschaft zu gute kommt.

Für diese Auffassung, dass die Mastzellen zu den Ernährungsverbältnissen des Nerven in der innigsten Beziehung stehen, möchte ich nicht zum wenigsten die auffallende Thatsache, auf die ich schon oben binwies, geltend machen, dass diese Elemente nur in den Verzweigungen der interstitiellen Substanz, wo keine Gefässe mehr anzutreffen sind, immer unmittelbar an die Schwannsche Scheide gelehnt, gefunden wurden.

Für die Entstehung der Mastzellen aus ächten Bindegewebskörpern will ich endlich noch das Factum verwerthen, dass ich weder in der weissen Substanz des Rückenmarks, noch in der Hirnrinde bisher Mastzellen nachweisen konnte. Die Ranvier'schen Körper in der Schwann'schen Scheide, aus denen die Mastzellen ja auch hervorgehen können, halte ich histologisch den Zellen der Henleschen Schicht gleichwerthig.

Die diagnostische Verwerthung des Mastzellenbefundes im Nerven ergiebt sich aus meinen Untersuchungen mit Leichtigkeit. Das, was im Alter auf Grund der veränderten Ernährungsvẹrhältnisse noch physiologisch ist, muss bei jugendlichen Individuen als von 
der Normabweichend, als pathologisch angesehen werden, d. h. der Befund von Mastzellen überhaupt bei Kindern bis zu 5 Jahren, ferner die Anwesenheit zahlreicher Mastzellen bei Individuen in der Blüthe des Lebens (etwa bis 45 Jahre) dentet im Nerven stets auf eine Ernährungsstörung. So fasse ich z. B. die Vermehrung der Hastzellen in dem Falle von multipler Neuritis als unzweifelhaft pathologisch auf.

Weitere Untersuchungen werden ergeben, in wie weit wir für andere Nervenerkrankungen Mastzellenbefunde zu verwerthen im Stande sind.

Am Schlusse sei es mir gestattet, meinen hochverehrten Vorgesetzten Herrn Director Dr. Riess und Herrn Prof. Dr. Fürbringer, die mir in liebenswürdigster Weise das Material zu diesen Untersuchungen zur Verfügung stellten, bestens zu danken.

\section{Erklärung der Abbildungen. (Taf. XII.)}

Fig. I. Querschnitt durch einen N. ischiadicus. Doppelfärbung: Carmin-Ammoniak und Anilinwassergentianaviolett. Oelimmersion 1/12. Ocul. 0 . Seibert.

Fig. II. Längsschnitt durch einen N. ischiadicus. Färbung: Anilinwassergentianaviolett. Oelimmersion 1/12. Ocul. 0. Seibert. 

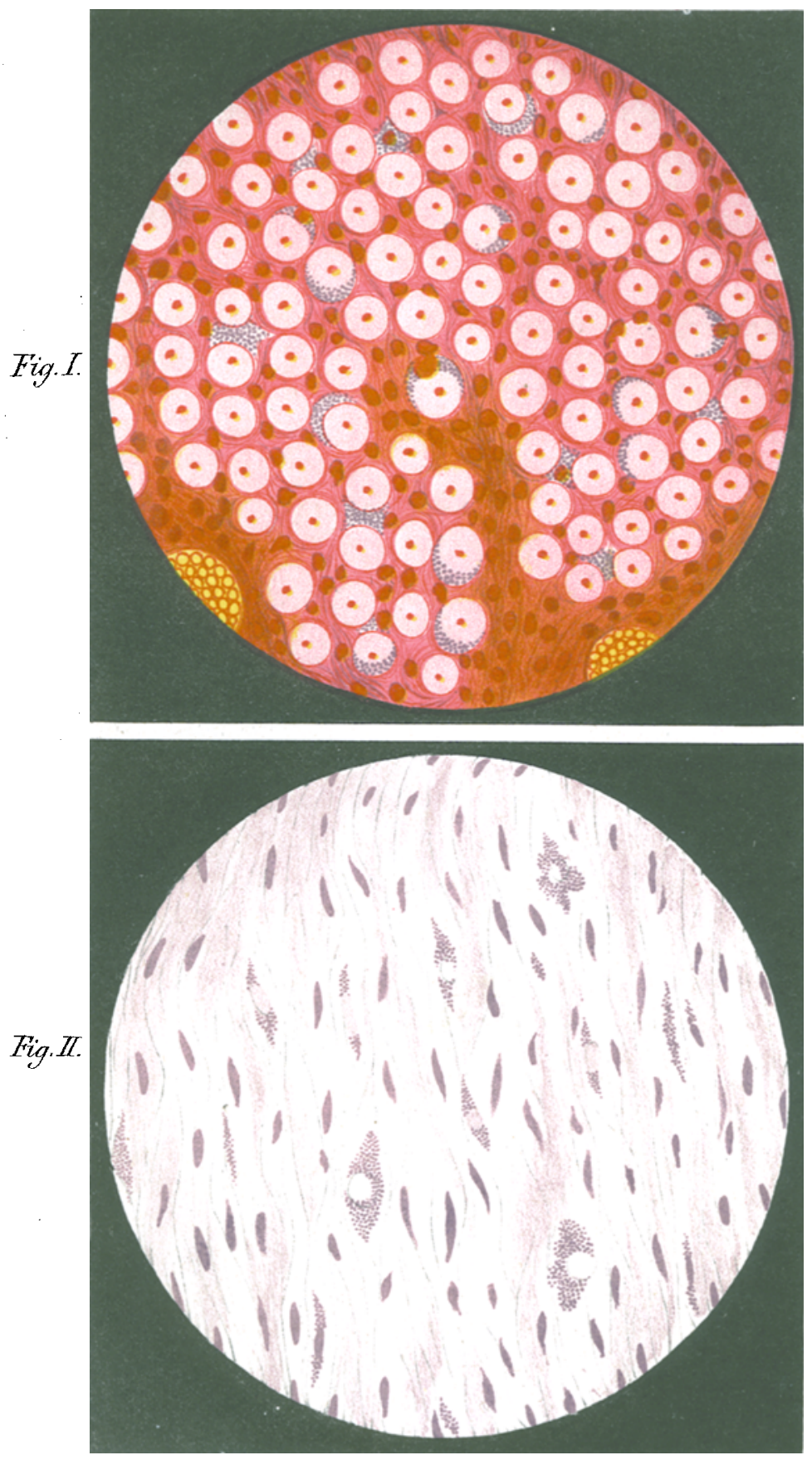